\title{
ON ASYMPTOTIC POINTWISE CONTRACTIONS IN METRIC SPACES
}

\author{
N. HUSSAIN, M.A. KHAMSI
}

\begin{abstract}
We discuss the existence of fixed points of asymptotic pointwise mappings in metric spaces. This is the nonlinear version of some known results proved in Banach spaces. We also discuss the case of multivalued mappings.

MSC: Primary 47H09; Secondary 47H10.

Keywords: Asymptotic pointwise contraction mapping, asymptotic pointwise nonexpansive mapping, multivalued mappings, fixed point, hyperconvex metric spaces, CAT(0) spaces, uniformly convex metric spaces.

\section{INTRODUCTION}

The notion of asymptotic pointwise mappings was introduced in [13, 14, 15]. The use of ultrapower technique was useful in proving some related fixed point results. In a recent paper [15], the authors gave simple and elementary proofs for the existence of fixed point theorems for asymptotic pointwise mappings without the use of ultrapowers. In this paper, we extend most of their results to metric spaces. In particular we consider the case of CAT(0) as an example of uniform convex metric spaces. In this work, we show how weak-compactness in Banach spaces is extended to metric spaces.
\end{abstract}

For more on metric fixed point theory, the reader may consult the book [12].

\section{Basic Definitions and Results}

First let us start by making some basic definitions. 
Definition 1. Let $(M, d)$ be a metric space. A mapping $T: M \rightarrow M$ is called a pointwise contraction if there exists a mapping $\alpha: M \rightarrow[0,1)$ such that

$$
d(T(x), T(y)) \leq \alpha(x) d(x, y) \text { for any } y \in M
$$

It is clear that pointwise contractive behavior was introduced to extend the contractive behavior in Banach contraction principle. The central fixed point result for such mappings is the following theorem

Theorem 2.1. ([13, 14]). Let $K$ be a weakly compact convex subset of a Banach space and suppose $T: K \rightarrow K$ is a pointwise contraction. Then $T$ has a unique fixed point, $x_{0}$. Moreover the orbit $\left\{T^{n}(x)\right\}$ converges to $x_{0}$, for each $x \in M$.

Note that if $T$ is a pointwise contraction, then it is continuous. Moreover if $\alpha(x)=0$ for some $x \in M$, then $T$ is a constant map. Since the main focus of this paper is about the fixed point problem, it is easy to prove that a pointwise contraction $T: M \rightarrow M$ has at most one fixed point, and if $a$ is its fixed point, then the orbit $\left\{T^{n}(x)\right\}$ converges to $a$, for each $x \in M$. Indeed, we have

$$
d\left(a, T^{n}(x)\right) \leq \alpha(a)^{n} d(a, x)
$$

for any $x \in M$. The above conclusion follows because $\alpha(a)<1$. It is not clear how to prove the existence of the fixed point from the convergence of the orbits which is the case in the classical proof given to the Banach contraction principle.

Definition 2. Let $(M, d)$ be a metric space. A mapping $T: M \rightarrow M$ is called an asymptotic pointwise mapping if there exists a sequence of mappings $\alpha_{n}: M \rightarrow$ $[0, \infty)$ such that

$$
d\left(T^{n}(x), T^{n}(y)\right) \leq \alpha_{n}(x) d(x, y) \text { for any } y \in M
$$

(i) If $\left\{\alpha_{n}\right\}$ converges pointwise to $\alpha: M \rightarrow[0,1)$, then $T$ is called an asymptotic pointwise contraction.

(ii) If $\limsup _{n \rightarrow \infty} \alpha_{n}(x) \leq 1$, then $T$ is called asymptotic pointwise nonexpansive.

(ii) If $\limsup _{n \rightarrow \infty} \alpha_{n}(x) \leq k$, with $0<k<1$, then $T$ is called strongly asymptotic pointwise contraction. 


\section{Pointwise Contractions in Metric Spaces}

Let $M$ be a metric space and $\mathcal{F}$ a family of subsets of $M$. Then we say that $\mathcal{F}$ defines a convexity structure on $M$ if it contains the closed balls and is stable by intersection. For instance $\mathcal{A}(M)$, the class of the admissible subsets of $M$, defines a convexity structure on any metric space $M$. Recall that a subset of $M$ is admissible if it is a nonempty intersection of closed balls.

At this point we introduce some notation which will be used throughout the remainder of this work. For a subset $A$ of a metric space $M$, set:

$$
\begin{aligned}
& r_{x}(A) \quad=\sup \{d(x, y): y \in A\}, \quad x \in M ; \\
& R(A) \quad=\inf \left\{r_{x}(A): x \in A\right\} \text {; } \\
& \operatorname{diam}(A)=\sup \{d(x, y): x, y \in A\} ; \\
& C_{A}(A)=\left\{x \in A: r_{x}(A)=R(A)\right\} \text {; } \\
& \operatorname{cov}(A)=\bigcap\{B: B \text { is a ball and } B \supseteq A\} \text {. }
\end{aligned}
$$

$\operatorname{diam}(A)$ is called the diameter of $A, R(A)$ is called the Chebyshev radius of $A$, $C_{A}(A)$ is called the Chebyshev center of $A$, and $\operatorname{cov}(A)$ is called the cover of $A$.

Definition 3. Let $\mathcal{F}$ be a convexity structure on $M$.

(i) We will say that $\mathcal{F}$ is compact if any family $\left(A_{\alpha}\right)_{\alpha \in \Gamma}$ of elements of $\mathcal{F}$, has a nonempty intersection provided $\bigcap_{\alpha \in F} A_{\alpha} \neq \emptyset$ for any finite subset $F \subset \Gamma$.

(ii) We will say that $\mathcal{F}$ is normal if for any $A \in \mathcal{F}$, not reduced to one point, we have $R(A)<\operatorname{diam}(A)$.

(iii) We will say that $\mathcal{F}$ is uniformly normal if there exists $c \in(0,1)$ such that for any $A \in \mathcal{F}$, not reduced to one point, we have $R(A) \leq c \operatorname{diam}(A)$. It is easy to check that $c \geq 1 / 2$.

Example 1. A metric space $M$ is said to be hyperconvex [1] if given any family $\left\{x_{\alpha}\right\}$ of points of $M$ and any family $\left\{r_{\alpha}\right\}$ of nonnegative real numbers satisfying

$$
d\left(x_{\alpha}, x_{\beta}\right) \leq r_{\alpha}+r_{\beta}
$$


it is the case that $\cap_{\alpha} B\left(x_{\alpha} ; r_{\alpha}\right) \neq \emptyset$. It is well known $[2,7]$ that if $M$ is hyperconvex, then $\mathcal{A}(M)$ is compact and uniformly normal with

$$
R(A)=\frac{1}{2} \operatorname{diam}(A)
$$

for any $A \in \mathcal{A}(M)$.

The main result of $[13,14]$ may be stated in metric spaces as follows

Theorem 3.1. Let $M$ be a bounded metric space. Assume that the convexity structure $\mathcal{A}(M)$ is compact. Let $T: M \rightarrow M$ be a pointwise contraction. Then $T$ has a unique fixed point, $x_{0}$. Moreover the orbit $\left\{T^{n}(x)\right\}$ converges to $x_{0}$, for each $x \in M$.

Proof. Since $\mathcal{A}(M)$ is compact, there exists a minimal nonempty $K \in \mathcal{A}(M)$ such that $T(K) \subset K$. It is easy to check that $\operatorname{cov}(T(K))=K$. Let $a \in K$, then we have $K \subset B\left(a, r_{a}(K)\right)$. Since $T$ is pointwise contraction, there exists a mapping $\alpha: M \rightarrow[0,1)$ such that

$$
d(T(x), T(y)) \leq \alpha(x) d(x, y) \text { for any } y \in M .
$$

In particular, we have then $T(K) \subset B\left(T(a), \alpha(a) r_{a}(K)\right)$, which implies $\operatorname{cov}(T(K)) \subset$ $B\left(T(a), \alpha(a) r_{a}(K)\right)$. So $r_{T(a)}(K) \leq \alpha(a) r_{a}(K)$. This will force $\operatorname{diam}(K)=0$. Indeed let $a \in K$ and define

$$
K_{a}=\left\{x \in K ; r_{x}(K) \leq r_{a}(K)\right\} .
$$

Clearly $K_{a}$ is not empty. Moreover we have

$$
K_{a}=\bigcap_{x \in K} B\left(x, r_{a}(K)\right) \cap K \in \mathcal{A}(M)
$$

And since $r_{T(a)}(K) \leq \alpha(a) r_{a}(K)$, we get $T\left(K_{a}\right) \subset K_{a}$. The minimality behavior of $K$ implies $K_{a}=K$. In particular we have $r_{x}(K)=r_{a}(K)$ for any $x \in K$. Hence $\operatorname{diam}(K)=r_{a}(K)$, for any $a \in K$, i.e. $a$ is a diametral point of $K$. Hence $\operatorname{diam}(K) \leq \alpha(a) \operatorname{diam}(K)$. And since $\alpha(a)<1$, we get $\operatorname{diam}(K)=0$, i.e. $K$ is reduced to one point which is fixed by $T$. Hence the fixed point set of $T$ is not empty. The remaining conclusion of the theorem follows from the general properties of pointwise contractions. 
It is well known $[2,7]$ that if $M$ is hyperconvex, then $\mathcal{A}(M)$ is compact, hence we obtain:

Corollary 1. Let $M$ be a bounded hyperconvex metric space. Let $T: M \rightarrow M$ be a pointwise contraction. Then $T$ has a unique fixed point, $x_{0}$. Moreover the orbit $\left\{T^{n}(x)\right\}$ converges to $x_{0}$, for each $x \in M$.

\section{Asymptotically Pointwise Contractions}

Let $M$ be a metric space and $\mathcal{F}$ a convexity structure. We will say that a function $\Phi: M \rightarrow[0, \infty)$ is $\mathcal{F}$-convex if $\{x ; \Phi(x) \leq r\} \in \mathcal{F}$ for any $r \geq 0$. Also we define a type to be a function $\Phi: M \rightarrow[0, \infty)$ defined as

$$
\Phi(u)=\limsup _{n \rightarrow \infty} d\left(x_{n}, u\right)
$$

where $\left(x_{n}\right)$ is a bounded sequence in $M$. Types are very useful in the study of the geometry of Banach spaces and the existence of fixed point of mappings. We will say that a convexity structure $\mathcal{F}$ on $M$ is T-stable if types are $\mathcal{F}$-convex. We have the following lemma.

Lemma 4.1. Let $M$ be a metric space and $\mathcal{F}$ a compact convexity structure on $M$ which is T-stable. Then for any type $\Phi$, there exists $x_{0} \in M$ such that

$$
\Phi\left(x_{0}\right)=\inf \{\Phi(x) ; x \in M\} .
$$

The proof is easy and will be omitted.

Theorem 4.1. Let $M$ be a bounded metric space. Assume that the convexity structure $\mathcal{A}(M)$ is compact. Let $T: M \rightarrow M$ be a strongly asymptotic pointwise contraction. Then $T$ has a unique fixed point, $x_{0}$. Moreover the orbit $\left\{T^{n}(x)\right\}$ converges to $x_{0}$, for each $x \in M$.

Proof. First note that $T$ has at most one fixed point. Indeed, let $a, b \in M$ be two fixed points of $T$. Then we have

$$
d(a, b)=d\left(T^{n}(a), T^{n}(b)\right) \leq \alpha_{n}(a) d(a, b) .
$$

If we let $n$ go to infinity, we get $d(a, b) \leq k d(a, b)$ for some $k \in(0,1)$. This will force $d(a, b)=0$. Next let $x \in M$ and define the type

$$
\Phi(u)=\limsup _{n \rightarrow \infty} d\left(T^{n}(x), u\right), \text { for each } u \in M .
$$


Since $\mathcal{A}(M)$ is compact, then

$$
\Omega(x)=\bigcap_{n \geq 1} \operatorname{cov}\left(\left\{T^{k}(x) ; k \geq n\right\}\right) \neq \emptyset .
$$

Let $\omega \in \Omega(x)$. Then we have

$$
d\left(T^{m+n+h}(x), T^{m+h}(x)\right) \leq \alpha_{h}\left(T^{m}(x)\right) d\left(T^{n}(x), T^{m}(x)\right) .
$$

If we let $n$ go to infinity, we get

$$
\Phi\left(T^{m+h}(x)\right) \leq \alpha_{h}\left(T^{m}(x)\right) \Phi\left(T^{m}(x)\right) .
$$

Next we let $h$ go to infinity to get

$$
\limsup _{n \rightarrow \infty} \Phi\left(T^{n}(x)\right) \leq k \Phi\left(T^{n}(x)\right)
$$

for some $k \in(0,1)$, which easily implies that $\limsup _{n \rightarrow \infty} \Phi\left(T^{n}(x)\right)=0$. Next we notice that

$$
\Phi(\omega) \leq \limsup _{n \rightarrow \infty} \Phi\left(T^{n}(x)\right)=0 .
$$

Indeed let $u \in M$, then for any $\varepsilon>0$, then there exists $n_{0} \geq 1$ such that for any $n \geq n_{0}$

$$
d\left(T^{n}(x), u\right) \leq \Phi(u)+\varepsilon .
$$

In particular we have $T^{n}(x) \in B(u, \Phi(u)+\varepsilon)$, for any $n \geq n_{0}$. So

$$
\Omega(x) \subset \operatorname{cov}\left(\left\{T^{n}(x) ; n \geq n_{0}\right\}\right) \subset B(u, \Phi(u)+\varepsilon),
$$

which implies $\omega \in B(u, \Phi(u)+\varepsilon)$. This is true for any $\varepsilon>0$. Hence for any $u \in M$ we have $d(\omega, u) \leq \Phi(u)$. Hence

$$
\Phi(\omega)=\limsup _{n \rightarrow \infty} d\left(T^{n}(x), \omega\right) \leq \limsup _{n \rightarrow \infty} \Phi\left(T^{n}(x)\right) .
$$

Therefore we have $\Phi(\omega)=0$ which implies that $\left\{T^{n}(x)\right\}$ converges to $\omega$. This will force $\omega$ to be a fixed point of $T$. Since we already noticed that $T$ has at most one fixed point, then $T$ has a fixed point $x_{0}$ and any orbit converges to $x_{0}$.

If $M$ is hyperconvex, then $\mathcal{A}(M)$ is compact, hence we obtain: 
Corollary 2. Let $M$ be a bounded hyperconvex metric space. Let $T: M \rightarrow M$ be a strongly asymptotic pointwise contraction. Then $T$ has a unique fixed point, $x_{0}$. Moreover the orbit $\left\{T^{n}(x)\right\}$ converges to $x_{0}$, for each $x \in M$.

Next we relax the strong behavior of $T$ but assume that types are convex to obtain the following result

Theorem 4.2. Let $M$ be a bounded metric space. Assume that there exists a convexity structure $\mathcal{F}$ which is compact and T-stable. Let $T: M \rightarrow M$ be an asymptotic pointwise contraction. Then $T$ has a unique fixed point, $x_{0}$. Moreover the orbit $\left\{T^{n}(x)\right\}$ converges to $x_{0}$, for each $x \in M$.

Proof. Similarly one can easily show that $T$ has at most one fixed point. As we did in the proof of the previous result, let $x \in M$ and define the type

$$
\Phi(u)=\limsup _{n \rightarrow \infty} d\left(T^{n}(x), u\right), \text { for each } u \in M .
$$

Since $\mathcal{F}$ is compact and T-stable, then there exists $x_{0} \in M$ such that

$$
\Phi\left(x_{0}\right)=\inf \{\Phi(u) ; u \in M\} .
$$

Let us show that $\Phi\left(x_{0}\right)=0$. Indeed we have

$$
d\left(T^{n+m}(x), T^{m}\left(x_{0}\right)\right) \leq \alpha_{m}\left(x_{0}\right) d\left(T^{n}(x), x_{0}\right),
$$

for any $n, m \geq 1$. If we let $n$ go to infinity, we get

$$
\Phi\left(T^{m}\left(x_{0}\right)\right) \leq \alpha_{m}\left(x_{0}\right) \Phi\left(x_{0}\right)
$$

which implies

$$
\Phi\left(x_{0}\right)=\inf \{\Phi(u) ; u \in M\} \leq \Phi\left(T^{m}\left(x_{0}\right)\right) \leq \alpha_{m}\left(x_{0}\right) \Phi\left(x_{0}\right) .
$$

If we let $m$ go to infinity, we get $\Phi\left(x_{0}\right) \leq \alpha\left(x_{0}\right) \Phi\left(x_{0}\right)$. Since $\alpha\left(x_{0}\right)<1$, we get $\Phi\left(x_{0}\right)=0$, which implies that $\left\{T^{n}(x)\right\}$ converges to $x_{0}$. This will force $x_{0}$ to be a fixed point of $T$. Since we already noticed that $T$ has at most one fixed point, then $T$ has a fixed point $x_{0}$ and any orbit converges to $x_{0}$.

\section{Asymptotic Pointwise Nonexpansive mappings}

We should note that any result on asymptotic pointwise nonexpanisve mappings in metric spaces should extend what is known on asymptotic nonexpansive mappings in metric spaces. Unfortunately only a partial result in hyperconvex 
metric spaces is known (see [11]). Here we extend the result found in [15] in uniformly Banach spaces to CAT(0) metric spaces. Indeed these metric spaces offer a nice example of uniformly convex metric spaces. It is not clear that the main inequality used in [15] satisfied in uniformly convex Banach spaces has a similar one in uniformly convex metric spaces.

A metric space $(X, d)$ is said to be a length space if each two points of $X$ are joined by a rectifiable path (that is, a path of finite length) and the distance between any two points of $X$ is taken to be the infimum of the lengths of all rectifiable paths joining them. In this case, $d$ is said to be a length metric (otherwise known an inner metric or intrinsic metric). In case no rectifiable path joins two points of the space the distance between them is said to be $\infty$.

A geodesic path joining $x \in X$ to $y \in X$ (or, more briefly, a geodesic from $x$ to $y)$ is a map $c$ from a closed interval $[0, l] \subset \mathbb{R}$ to $X$ such that $c(0)=x, c(l)=y$, and $d\left(c(t), c\left(t^{\prime}\right)\right)=\left|t-t^{\prime}\right|$ for all $t, t^{\prime} \in[0, l]$. In particular, $c$ is an isometry and $d(x, y)=l$. The image $\alpha$ of $c$ is called a geodesic (or metric) segment joining $x$ and $y .(X, d)$ is said to be a geodesic space if every two points of $X$ are joined by a geodesic. $X$ is said to be uniquely geodesic if there is exactly one geodesic joining $x$ and $y$ for each $x, y \in X$, which we will denote by $[x, y]$ called the segment joining $x$ to $y$.

A geodesic triangle $\Delta\left(x_{1}, x_{2}, x_{3}\right)$ in a geodesic metric space $(X, d)$ consists of three points in $X$ (the vertices of $\Delta$ ) and a geodesic segment between each pair of vertices (the edges of $\Delta$ ). A comparison triangle for geodesic triangle $\Delta\left(x_{1}, x_{2}, x_{3}\right)$ in $(X, d)$ is a triangle $\bar{\Delta}\left(x_{1}, x_{2}, x_{3}\right):=\Delta\left(\bar{x}_{1}, \bar{x}_{2}, \bar{x}_{3}\right)$ in $M_{\kappa}^{2}$ such that $d_{\mathbb{R}^{2}}\left(\bar{x}_{i}, \bar{x}_{j}\right)=d\left(x_{i}, x_{j}\right)$ for $i, j \in\{1,2,3\}$. If $\kappa>0$ it is further assumed that the perimeter of $\Delta\left(x_{1}, x_{2}, x_{3}\right)$ is less than $2 D_{\kappa}$, where $D_{\kappa}$ denotes the diameter of $M_{\kappa}^{2}$. Such a triangle always exists.

A geodesic metric space is said to be a $\operatorname{CAT}(\kappa)$ space if all geodesic triangles of appropriate size satisfy the following $\mathrm{CAT}(\kappa)$ comparison axiom.

CAT $(\kappa)$ : Let $\Delta$ be a geodesic triangle in $X$ and let $\bar{\Delta} \subset M_{\kappa}^{2}$ be a comparison triangle for $\Delta$. Then $\Delta$ is said to satisfy the $\operatorname{CAT}(\kappa)$ inequality if for all $x, y \in \Delta$ 
and all comparison points $\bar{x}, \bar{y} \in \bar{\Delta}$,

$$
d(x, y) \leq d(\bar{x}, \bar{y}) .
$$

Complete $\mathrm{CAT}(0)$ spaces are often called Hadamard spaces. These spaces are of particular relevance to this study.

Finally we observe that if $x, y_{1}, y_{2}$ are points of a CAT(0) space and if $y_{0}$ is the midpoint of the segment $\left[y_{1}, y_{2}\right]$, which we will denote by $\frac{y_{1} \oplus y_{2}}{2}$, then the CAT(0) inequality implies

$$
d\left(x, \frac{y_{1} \oplus y_{2}}{2}\right)^{2} \leq \frac{1}{2} d\left(x, y_{1}\right)^{2}+\frac{1}{2} d\left(x, y_{2}\right)^{2}-\frac{1}{4} d\left(y_{1}, y_{2}\right)^{2}
$$

because equality holds in the Euclidean metric. In fact (see [3], p. 163), a geodesic metric space is a CAT(0) space if and only if it satisfies inequality (1) (which is known as the $\mathrm{CN}$ inequality of Bruhat and Tits [4]). Moreover if $M$ is a $\operatorname{CAT}(0)$ metric space and $x, y \in M$, then for any $\alpha \in[0,1]$ there exists a unique point $\alpha x \oplus(1-\alpha) y \in[x, y]$ such that

$$
d(z, \alpha x \oplus(1-\alpha) y) \leq \alpha d(z, x)+(1-\alpha) d(z, y), \text { for any } z \in M,
$$

and $[x, y]=\{\alpha x \oplus(1-\alpha) y, \alpha \in[0,1]\}$.

Let $M$ be a complete $\mathrm{CAT}(0)$ space. A subset $C \subset M$ is convex if for any $x, y \in C$ we have $[x, y] \subset C$. Denote by $\mathcal{C}(M)$ the family of all closed convex subsets of $M$. Then $\mathcal{C}(M)$ defines a convexity structure which is compact and uniformly normal [6]. Note that any type function is convex, i.e. $\mathcal{C}(M)$ is Tconvex. This follows easily from the inequality (2). A direct implication of these properties is that any type function achieves its infinimum, i.e. for any bounded sequence $\left\{x_{n}\right\}$ in a $\operatorname{CAT}(0)$ space $M$, there exists $\omega \in M$ such that $\Phi(\omega)=\inf \{\Phi(x) ; x \in M\}$, where

$$
\Phi(x)=\limsup _{n \rightarrow \infty} d\left(x_{n}, x\right) .
$$


Theorem 5.1. Let $M$ be a complete $C A T(0)$ metric space. Let $C$ be a bounded closed nonempty convex subset of $M$. Then any $T: C \rightarrow C$ pointwise asymptotically nonexpansive has a fixed point. The fixed point set Fix $(T)$ is closed and convex, i.e. $\operatorname{Fix}(T) \in \mathcal{C}(M)$.

Proof. As before, let $x \in C$ and define the type

$$
\Phi(u)=\limsup _{n \rightarrow \infty} d\left(T^{n}(x), u\right), \text { for any } u \in C .
$$

Let $\omega \in C$ such that $\Phi(\omega)=\inf \{\Phi(u) ; u \in C\}=\Phi_{0}$. We have seen that $\Phi\left(T^{n}(\omega)\right) \leq \alpha_{n}(\omega) \Phi(\omega)=\alpha_{n}(\omega) \Phi_{0}$, for any $n \geq 1$. The CN inequality implies

$$
\begin{aligned}
d\left(T^{n}(x), \frac{T^{m}(\omega) \oplus T^{h}(\omega)}{2}\right)^{2} \leq & \frac{1}{2} d\left(T^{n}(x), T^{m}(\omega)\right)^{2}+\frac{1}{2} d\left(T^{n}(x), T^{h}(\omega)\right)^{2} \\
& -\frac{1}{4} d\left(T^{m}(\omega), T^{h}(\omega)\right)^{2} .
\end{aligned}
$$

If we let $n$ go to infinity, we get

$\Phi_{0}^{2} \leq \Phi\left(\frac{T^{m}(\omega) \oplus T^{h}(\omega)}{2}\right)^{2} \leq \frac{1}{2} \Phi\left(T^{m}(\omega)\right)^{2}+\frac{1}{2} \Phi\left(T^{h}(\omega)\right)^{2}-\frac{1}{4} d\left(T^{m}(\omega), T^{h}(\omega)\right)^{2}$

which implies

$$
d\left(T^{m}(\omega), T^{h}(\omega)\right)^{2} \leq \Phi_{0}^{2}\left(2 \alpha_{m}(\omega)^{2}+2 \alpha_{h}^{2}(\omega)-4\right)
$$

Since $T$ is pointwise asymptotic nonexpansive, we get

$$
\limsup _{m, h \rightarrow \infty}\left(T^{m}(\omega), T^{h}(\omega)\right)^{2} \leq 0
$$

which implies $\left\{T^{n}(\omega)\right\}$ is a Cauchy sequence. Let $v=\lim _{n \rightarrow \infty} T^{n}(\omega)$. Since $T$ is continuous, then $T(v)=v$, i.e. $v$ is a fixed point of $T$. This proves that $\operatorname{Fix}(T)$ is not empty. Again since $T$ is continuous, Fix $(T)$ is closed. In order to prove that $\operatorname{Fix}(T)$ is convex, it is enough to prove that $\frac{x \oplus y}{2} \in \operatorname{Fix}(T)$, whenever $x, y \in F i x(T)$. Indeed set $\omega=\frac{x \oplus y}{2}$. The CN inequality implies

$$
d\left(T^{n}(\omega), \omega\right)^{2} \leq \frac{1}{2} d\left(x, T^{n}(\omega)\right)^{2}+\frac{1}{2} d\left(y, T^{n}(\omega)\right)^{2}-\frac{1}{4} d(x, y)^{2}
$$


for any $n \geq 1$. Since

$$
d\left(x, T^{n}(\omega)\right)^{2}=d\left(T^{n}(x), T^{n}(\omega)\right)^{2} \leq \alpha_{n}^{2}(\omega) d(\omega, x)^{2}=\frac{\alpha_{n}^{2}(\omega) d(x, y)^{2}}{4},
$$

and

$$
d\left(y, T^{n}(\omega)\right)^{2}=d\left(T^{n}(y), T^{n}(\omega)\right)^{2} \leq \alpha_{n}^{2}(\omega) d(\omega, y)^{2}=\frac{\alpha_{n}^{2}(\omega) d(x, y)^{2}}{4}
$$

we get

$$
d\left(T^{n}(\omega), \omega\right)^{2} \leq \frac{\left(\alpha_{n}^{2}(\omega)-1\right) d(x, y)^{2}}{4},
$$

for any $n \geq 1$. Since $T$ is pointwise asymptotic nonexpansive, we get $\lim _{n \rightarrow \infty} T^{n}(\omega)=$ $\omega$, which implies $T(\omega)=\omega$, i.e. $\omega \in \operatorname{Fix}(T)$.

If $U, V$ are bounded subsets of a metric space $X$, let $H$ denote the Hausdorff metric defined as usual by

$$
H(U, V)=\inf \left\{\varepsilon>0: U \subset N_{\varepsilon}(V) \text { and } V \subset N_{\varepsilon}(U)\right\},
$$

where $N_{\varepsilon}(V)=\{y \in X: d(y, V)<\varepsilon\}$. Let $E$ be a subset of a metric space $X$. A mapping $T: E \rightarrow 2^{X}$ with nonempty bounded values is nonexpansive provided $H(T(x), T(y)) \leq d(x, y)$ for all $x, y \in E$. Let $t: E \rightarrow E$ and $T: E \rightarrow 2^{X}$ with $T(x) \cap E \neq \emptyset$ for $x \in E$. Then $t$ and $T$ are said to be commuting mappings if $t(y) \in T(t(x)) \cap E$ for all $y \in T(x) \cap E$ and for all $x \in E$. A point $z \in X$ is called a center [8] for a mapping $t: E \rightarrow X$ if for each $x \in E, d(z, t(x)) \leq d(z, x)$. The set $Z(t)$ denotes the set of all centers of the mapping $t$.

As an application of our Theorem 5.1 we obtain:

Theorem 5.2. Let $M$ be a complete $C A T(0)$ space and $C$ be a bounded closed convex subset of $M$. Assume $t: C \rightarrow C$ is pointwise asymptotically nonexpansive and $T: C \rightarrow 2^{C}$ is nonexpansive mappings with $T(x)$ a compact convex subset of $C$ for each $x \in C$. If the mappings $t$ and $T$ commute and satisfy the condition

$$
T(x) \cap F i x(t) \subset Z(t), \quad \text { for all } x \in F i x(t)
$$

then there is $z \in C$ such that $z=t(z) \in T(z)$. 
Proof. By Theorem 5.1, a pointwise asymptotically nonexpansive self-mapping $t$ of a bounded closed convex subset has a nonempty fixed point set $A$ which is a closed convex subset of $M$. Since $t$ and $T$ commute, $t(y) \in T(t(x))=T(x)$ for $y \in T(x)$ and $x \in A$, and therefore, $T(x)$ is invariant under $t$ for each $x \in A$. Since $T(x)$ is a closed bounded convex subset of a $C A T(0)$ space, $t$ has a fixed point in $T(x)$ and $T(x) \cap A \neq \emptyset$ for $x \in A$. Now consider the mapping $T(.) \cap A: A \rightarrow$ compact convex subsets of $A$. We claim that this mapping is nonexpansive. Indeed, if $u \in T(x) \cap A$ for some $x \in A$, let $v$ be the unique closest point in $T(y)$ to $u$ for some $y \in A$. Then $d(u, v)=\inf \{d(u, w) ; w \in T(y)\}$.

However, since $u \in Z(t), d(u, t(v)) \leq d(u, v)$, which contradicts the uniqueness of $v$ as the closest point to $u$. Therefore, $v=t(v)$ implying $v \in T(y) \cap A$. Since this argument is symmetric in the points $x$ and $y$, it follows that

$$
H(T(x) \cap A, T(y) \cap A) \leq H(T(x), T(y)) \leq d(x, y) \text { for } \quad x, y \in A .
$$

By [10] the nonexpansive mapping $T(.) \cap A: A \rightarrow$ compact convex subsets of $A$ has a fixed point $z \in T(z) \cap A$. Therefore, $z=t(z) \in T(z)$.

The Theorem 5.2 may be seen as an extension to Theorem 4.1 of [5].

Corollary 3. Let $M$ be a complete $C A T(0)$ space and $C$ be a bounded closed convex subset of $M$. Assume $t: C \rightarrow C$ is pointwise asymptotically nonexpansive and $T: C \rightarrow 2^{C}$ is nonexpansive mappings with $T(x)$ a compact convex subset of $C$ for each $x \in C$. If the mappings $t$ and $T$ satisfy the condition

$$
\emptyset \neq T(x) \cap F i x(t) \subset Z(t), \text { for all } x \in F i x(t)
$$

then there is $z \in C$ such that $z=t(z) \in T(z)$.

A close look at the above proof suggests that a pointwise asymptotic mapping in CAT(0) metric space is may be demi-closed as it was noticed by Göhde [9] for nonexpanisve mapping in uniformly Banach spaces. Before we state the next and final result of this work, we need the following notation

$$
\left\{x_{n}\right\} \rightarrow \omega \text { if and only if } \Phi(\omega)=\inf _{x \in C} \Phi(x),
$$


where $C$ is a closed convex subset which contains the bounded sequence $\left\{x_{n}\right\}$ and $\Phi(x)=\limsup _{n \rightarrow \infty} d\left(x_{n}, x\right)$.

Proposition 1. Let $M$ be a $C A T(0)$ metric space. Let $C$ be a bounded closed nonempty convex subset of $M$. Let $T: C \rightarrow C$ be a pointwise asymptotic nonexpansive mapping. Let $\left\{x_{n}\right\} \in C$ be an approximate fixed point sequence, i.e. $\lim _{n \rightarrow \infty} d\left(x_{n}, T\left(x_{n}\right)\right)=0$, and $\left\{x_{n}\right\} \rightarrow \omega$. Then we have $T(\omega)=\omega$.

Proof. Since $\left\{x_{n}\right\}$ is an approximate fixed point sequence, then we have

$$
\Phi(x)=\limsup _{n \rightarrow \infty} d\left(T^{m}\left(x_{n}\right), x\right)
$$

for any $m \geq 1$. Hence $\Phi\left(T^{m}(x)\right) \leq \alpha_{m}(x) \Phi(x)$, for each $x \in C$. In particular, we have $\lim _{m \rightarrow \infty} \Phi\left(T^{m}(\omega)\right)=\Phi(\omega)$. The CN inequality implies

$$
d\left(x_{n}, \frac{\omega \oplus T^{m}(\omega)}{2}\right)^{2} \leq \frac{1}{2} d\left(x_{n}, \omega\right)^{2}+\frac{1}{2} d\left(x_{n}, T^{m}(\omega)\right)^{2}-\frac{1}{4} d\left(\omega, T^{m}(\omega)\right)^{2}
$$

for any $n, m \geq 1$. If we let $n \rightarrow \infty$, we will get

$$
\Phi\left(\frac{\omega \oplus T^{m}(\omega)}{2}\right)^{2} \leq \frac{1}{2} \Phi(\omega)^{2}+\frac{1}{2} \Phi\left(T^{m}(\omega)\right)^{2}-\frac{1}{4} d\left(\omega, T^{m}(\omega)\right)^{2}
$$

for any $m \geq 1$. The definition of $\omega$ implies

$$
\Phi(\omega)^{2} \leq \frac{1}{2} \Phi(\omega)^{2}+\frac{1}{2} \Phi\left(T^{m}(\omega)\right)^{2}-\frac{1}{4} d\left(\omega, T^{m}(\omega)\right)^{2}
$$

for any $m \geq 1$, or

$$
d\left(\omega, T^{m}(\omega)\right)^{2} \leq 2 \Phi\left(T^{m}(\omega)\right)^{2}-2 \Phi(\omega)^{2}
$$

If we let $m \rightarrow \infty$, we will get $\lim _{m \rightarrow \infty} d\left(\omega, T^{m}(\omega)\right)=0$. Hence $T(\omega)=\omega$ since $T$ is continuous.

\section{REFERENCES}

[1] N. Aronszajn, and P. Panitchpakdi, Extension of uniformly continuous transformations and hyperconvex metric spaces, Pacific J. Math. 6 (1956), 405-439.

[2] J. B. Baillon, Nonexpansive mappings and hyperconvex spaces, Contemp. Math. 72 (1988), 11-19. 
[3] M. Bridson and A. Haefliger, Metric spaces of non-positive curvature, Springer-Verlag, Berlin, Heidelberg, New York, 1999.

[4] F. Bruhat, and J. Tits, Groupes réductifs sur un corps local. I. Données radicielles valuées, Inst. Hautes Études Sci. Publ. Math. 41 (1972), 5-251.

[5] S. Dhompongsa, A. Kaewkhao, and B. Panayanak, Lims theorem for multivalued mappings in CAT(0) spaces, J. Math. Anal. Appl., 312 (2005), 478-487.

[6] S. Dhompongsa, , W.A. Kirk, , and Brailey Sims, Fixed points of uniformly lipschitzian mappings, Nonlinear Analysis, 65 (2006), 762-772.

[7] R. Espinola, and M.A. Khamsi, Introduction to hyperconvex spaces, Handbook of Metric Fixed Point Theory, W.A. Kirk and B. Sims, Editors, Kluwer Academic Publishers, 2001.

[8] J.Garcia-Falset, E.L.Fuster, and S.Prus, The fixed point property for mappings admitting a center, Nonlinear Analysis, 66 (2007), 1257-1274.

[9] Gohde D., Zum Prinzip der kontraktiven Abbildung, Math. Nachr. 30 (1965), 251-258.

[10] A. Kaewcharoen, and W.A. Kirk, Proximinality in geodesic spaces, Abstr. Appl. Anal., (2006) 1-10.

[11] M. A. Khamsi, On Asymptotically Nonexpansive Mappings in Hyperconvex Metric Spaces, Proceedings of the American Mathematical Society 132 (2004), 365-373.

[12] M. A. Khamsi, and W. A. Kirk, An Introduction to Metric Spaces and Fixed Point Theory, John Wiley, New York, 2001.

[13] W.A. Kirk, Fixed points of asymptotic contractions, J. Math. Anal. Appl. 277 (2003), 645650 .

[14] W.A. Kirk, Asymptotic pointwise contractions, in: Plenary Lecture, the 8th International Conference on Fixed Point Theory and Its Applications, Chiang Mai University, Thailand, July 16-22, 2007.

[15] W. A. Kirk, and Hong-Kun Xu, Asymptotic pointwise contractions, Nonlinear Analysis, 69 (2008), 4706-4712.

N. Hussain, Department of Mathematics, King Abdul Aziz University, PO Box 80203, JEDDAH 21589, SAUDi ARABIA.

E-mail address: nhusain@kau.edu.sa

M. A. Khamsi, Department of Mathematical Science, The University of Texas AT El Paso, El Paso, TX 79968, U.S.A.

E-mail address: mohamed@math.utep.edu 